\title{
A Review On Evaluation And Benefits Of Decision Support Systems
}

Roger Alan Pick, University of Missouri-Kansas City, USA

Nancy Weatherholt, University of Missouri-Kansas City, USA

\begin{abstract}
This review paper identifies benefits from various Decision Support Systems (DSSs) and introduces a range of examples from the literature. Those examples are organized according to the driving technological architecture of the DSS. Benefits may impact a decision or the decisionmaking process. Some techniques for assessing the benefits of a particular DSS were also surveyed.
\end{abstract}

Keywords: Improving Decision-Making; Decision Support System; DSS Benefits; Information System Assessment; Information System Benefits; Information System Evaluation

\section{INTRODUCTION}

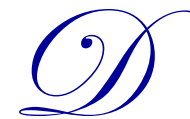

ecision support systems (DSS) now transcend any particular academic discipline. Computer-based decision support is used and taught in all business functional areas, many engineering fields, health care, and many other domains. In addition to its multiple areas of application, DSS today also exhibit a wide range of driving technologies.

\section{DSS Defined}

A Decision Support System (DSS) is "a computer-based system that supports choice by assisting the decision-maker in the organization of information and the modeling of outcomes." (Sauter, 2010, page 13) There are two essential ways that DDS is distinguished from other computer-based systems - first, a focus on decisionmaking and second, an interactive usage mode requiring both the system and the decision-maker to contribute to the decision-making process. A DSS is most useful in the context of semi-structured and unstructured problems. If the decision is made by the system without interaction with a decision-maker, the system is not a DSS; it is something else - perhaps an expert system or a transaction processing system.

\section{DSS Categories}

This paper is organized as follows: After this introductory section, we will discuss the process for evaluating DSS benefits. That process varies according to whether we are evaluating a DSS yet to be built as part of a justification process or if we are evaluating a DSS that has been built. In the former case, the options for evaluation are necessarily limited because the system does not yet exist and can only be discussed in hypothetical terms. In the latter case, we have the option of using the DSS as part of the evaluation or to audit test cases in which it has been used. The evaluations section is followed by a section discussing benefits from DSS.

The paper gives examples of some of the types of DSSs and some of the benefits that have resulted from them. Those examples are organized according to the type of DSS where we are categorizing the DSS according to the type of underlying technology which is most dominant in the particular DSS. The type of DSS is based upon which element of the system is most significant, according to a framework from Power (2002). Power's framework categorizes DSS as being communications-driven, data-driven, document-driven, knowledge-driven, or modeldriven. To this set of categories, we add the category of graphics-driven for those DSS in which the driving element 
is a graphical display of data. As an example of a type of DSS in this latter category, we might include a DSS built upon data from a geographic information system that displays data spatially. We end with concluding remarks.

The contribution of this paper is that we illustrate some of the myriad of benefits that a DSS can achieve using concrete examples from the literature. We also discuss how to evaluate the benefits of a specific DSS. This paper is designed to help readers gain a better understanding of the range of domains, both practical and scholarly, where DSS are used today.

\section{DSS EVALUATION}

\section{What Is Evaluation?}

Evaluation is a broad concept. (Adelman, 1992) It is an iterative process with the purpose of assessing the overall value of a system. It is distinct from but subsumes both validation, determining whether the right system is built, and verification, ensuring the system is built to specifications. (Turban et al, 2011). The notion of what constitutes a successful system has been evolving (Petter et al, 2012) and therefore what an evaluation involves changes as well. See Hosack et al (2012) for a thoughtful view of the future of DSS and DSS research.

There are many different types of evaluations. When a cost-benefit analysis is conducted, outcomes are measured in dollars. In contrast, a cost-effectiveness evaluation measures outcomes in non-financial terms such as the time required to make a decision. . An evaluation of overall effectiveness assesses to what extent the system is effective in assisting the organization to reach its goals. Efficiency evaluations determine whether more output could be attained with no increase in inputs, or whether inputs could be reduced while maintaining the current level of output. A goals-based evaluation is often conducted, but goal-free evaluation can be done, in which the actual effects of the system on the decision-maker(s) are evaluated in the absence of what individuals say they want to achieve. Formative evaluation is done assuming system continuation, with the objective of seeing how the system can be improved. Summative evaluation considers whether a system should be continued and, if so, at what level (Patton, 1982).

Organizations intending to evaluate a DSS should be mindful of the distinction between the evaluation framework, which is a strategy used to organize the evaluation process, and evaluation methods, which are applied to evaluate specific goals and are subsumed within the evaluation framework (Antunes et al, 2012). The framework should specify who will be involved in the evaluation, whether the evaluation will be conducted in situ, the expected cost of the evaluation, associated timelines of the process, the accuracy of the tools used in the process, and how feedback will be handled. All of these items need to be outlined before the evaluation is underway. Additionally, care must be taken in the handling of evaluation content, which includes any data that will be collected, any interpretations made of the data, and any recommendations offered (Patton, 1982).

\section{Why Evaluate?}

When an organization invests in a DSS, the investment decision is based on the premise that the DSS will improve the organization's performance. Evaluation assists decision-makers in their ongoing intra-organizational allocation of resources. If the results of the evaluation indicate that decision-making and organizational performance are enhanced by the DSS, then resources will continue to be allocated to its development and utilization (Adelman, 1992). In some situations, however, formal evaluation might not be necessary. In an environment characterized by rapidly changing conditions, the evaluation process itself should be viewed as evolving and adaptive, such that feedback about the DSS can be provided on a near-continuous basis (Hallikainen \& Chen, 2005).

\section{What Is Being Evaluated?}

With respect to system evaluation, Sauter (2010) distinguishes between testing the system and testing system implementation. The concern in the former is technical appropriateness, while in the latter evaluation is concerned with overall usefulness of the system. 
To assess technical appropriateness, many aspects of the DSS can be tested in isolation. However, all possible contingencies can never be tested when working with a DSS since system designers cannot anticipate the complete range of uses to which users will subject the DSS. Furthermore, because DDSs are used for semistructured and unstructured problems, the true accuracy of any decisions that the user working with the system produces is generally difficult to assess (Rhee \& Rao, 2008).

Scott (1995) specifies three approaches that could be used to determine whether the system is an implementation success:

- $\quad$ Evaluator determines whether DSS helped the organization acquire resources or improve its usage of scarce resources (input focus).

- $\quad$ Evaluator measures the extent to which DSS improved organizational effectiveness (output focus).

- $\quad$ Evaluator establishes whether DSS provides an improved process for decision-making, with the attention on the fact that it is a support system (process focus).

Antunes et al (2012) argue that evaluation methods should be explicitly designed to account for the nuances associated with collaborative systems in contrast to general information systems. Evaluation of collaborative systems (including GDSS) should consider group dynamics, environmental context in which it is situated, and impact of the technology on the group's processes as well as tasks. The authors incorporate a model of human performance in organizational settings as proposed by Reason (2008). Situation and situation control dimensions combine to form three performance levels. The three levels and corresponding characteristics of interest in evaluation are:

- $\quad$ Role-based performance, wherein group activity is essentially a set of independent activities

$\circ$ Focus is on the individual

- Data gathered is about efficiency and usability

- $\quad$ Rule-based performance, in which group activities are perceived to be coordinated

$\circ$ Evaluation focus is on a mixture of individual and group

- Data gathered concerns productivity and conformity

- $\quad$ Knowledge-based performance, comprising interdependent tasks

$\circ$ Focus is on the organization and group levels

- Data collected is about interaction, participation, satisfaction, consensus, usefulness and cost reductions

\section{When Should Evaluation Be Done?}

Generally speaking, the purpose of the evaluation determines when it should be done. For example, evaluations can be done early in the development process as part of a cost-benefit study, justifying subsequent effort to create the DSS. Evaluations can also be done late in the development process as part of a software testing effort. Many academics conduct evaluations of the DSS in the field in order to document the quality of their scholarly contribution and thereby justify publication.

Based on the premise that DSS developers should use different evaluation approaches from traditional methods used by Information Systems (IS) developers, Miah et al (2012) suggest a DSS evaluation strategy grounded in a design science approach. Specifically, they implement a mental model approach that incorporates checkpoints in six phases of the development process as advocated by Peffers et al (2008). They assert that an evaluation strategy should be based within the problem domain context since "most DSS development problems result from poor identification of end users' needs." The six-phase checkpoints are: (1) Problem definitions; (2) Design objectives; (3) Artefact design and development; (4) Design context; (5) Effectiveness and efficiency measurements; and (6) Communication of results. The authors evaluate their interpretive approach using interviews with four individuals. Input from the targeted stakeholders suggests that all six phases are important components in 
the process. Communication of results is considered to be the most important phase. That being said, design context, as well as effectiveness and efficiency, are also viewed as very important by all respondents. While acknowledging the limited number of participants in the process, the authors contend they present an important developmentoriented approach to consider in future DSS evaluations.

In contrast, Antunes et al (2012) suggest a framework for evaluation based on product development stages broken down as: conception, implementation, production, reengineering, and procurement. Adelman (1992) states, "evaluation is an inherent part of the prototyping development approach." Turban et al (2011) indicate evaluation activities should be repeated whenever the prototype is changed. Similarly, Hallikainen \& Chen (2005) emphasize the importance of conducting regular evaluations throughout the system development process. Evaluation should be fed into a feedback loop, leading to corrective actions as applicable. Rennie \& Singh (1995) state, "effective evaluation is 'an on-going, parallel process which is indeed codependent, iterative and cyclic.'”

\section{Who Should Be Involved In The Evaluation Process?}

Ideally, evaluation includes a wide range of people. Hallikainen \& Chen (2005) indicate that all relevant stakeholders should be specified during evaluation. Rennie \& Singh (1995) emphasize that participatory evaluation is most effective. Jiggins (1995) states, "reality is not a given actuality waiting to be discovered by the detached scientist, but a constructed understanding -- an informed perception -- developed by those engaged in the activity under scrutiny." Because determining whether observed changes in a setting are caused by changed inputs often involves contextual interpretation, it is usually best to include personnel in the evaluation process who are in a position to be able to make appropriate conclusions.

An evaluation facilitator should know the system, the context in which it is (or will be) used, and the corporate culture. Additionally, he or she should have good conflict resolution skills, be flexible and adaptive, creative and able to work with individuals and in group settings. An evaluation team should include DSS users as well as individuals who will be affected by changes resulting from the system's use. People selected should be able to incorporate evaluation feedback to make improvements to the system or the environment in which it is used. They should believe that the evaluation is worthwhile, and they should be able to devote sufficient time to the evaluation process (Rennie \& Singh, 1995).

Ultimately, the focus of the evaluation process will be different depending on who initiates the process as well as who is conducting the evaluation. Evaluator knowledge and skills will ultimately determine whether organizational interests are appropriately considered in the evaluation process (Hallikainen \& Chen, 2005).

\section{How Is Evaluation Performed?}

The prototyping approach often used in decision support system development suggests the use of a multifaceted evaluation approach. Technical, empirical, and subjective facets comprise such an approach. Technical evaluation methods relate to system verification, whereas empirical evaluation methods assess validation. Subjective evaluation methods are applicable to both verification and validation, although they are used primarily in evaluating validation (Adelman, 1992). A number of recent studies are discussed below, indicating varied evaluation methods suggested and in use.

The Defense Acquisition Guidebook, Chapter 9 "Test and Evaluation," (Defense, Department of, 2012) provides details of the U.S. Department of Defense testing and evaluation strategy for the three primary decision support systems used to acquire materiel and services. An event-driven integrated strategy situated within the program's overall acquisition strategy is used. The author claims the process used results in resource efficiencies and an enhanced data set useful for separate evaluations. The Department bases the integrated strategy on an evaluation framework. This framework indicates the links between key program and user decisions. It also describes developmental and operational areas requiring evaluation related to those decisions. Key performance parameters, key system attributes, critical technical parameters, key test measures (measures of suitability and effectiveness), planned test methods, key test resources, facility and infrastructure needs are specified. Additionally, any limitations or major risks to completing the evaluations are identified. The author notes that it is important in evaluation 
framework discussions to provide concise descriptions of links between key decisions in the system life cycle, areas needing evaluation to support those decisions, and the test methods needed to get the data for the evaluations.

Examining research in the area of financial decision support, Alic et al (2012) indicate much of the focus falls into three areas: financial analysis (most common), risk management, and fraud detection. Most of the studies in their sample analyzed examined needs aspects of the DSS without explicitly involving the users. The authors point out that many evaluation methods, both quantitative and qualitative, are discussed in the design science literature as being appropriate, but all of the financial DSS studies they analyzed conducted an evaluation based on metrics such as precision, recall or accuracy, or based on simulation.

Barfod (2012) examines decision support in the realm of transport infrastructure assessments. Barfod suggests that in recent years, some organizational decisions need to incorporate certain key variables that are not socio-economic in nature, and assessment methods need to be used that can adequately capture the variables. Claiming that much prior research used simplistic cost-benefit analysis, the author proposes a composite model for assessment (COSIMA). The COSIMA DSS proposed uses multi-criteria decision analysis (MCDA) in conjunction with a cost-benefit analysis (required by regulatory authorities in some settings) to assess complex decision problems. The author suggests incorporating a decision conference including not only the decision-maker(s) but any other stakeholders who might interact with the DSS. This would constitute an intervention phase within the preliminary problem-structuring phase in which model building occurs. It would also allow for assessment revision to arrive at a shared understanding before the detailed analytic structure is derived.

Asserting that examining the information load in terms of alternatives provided is more important than the number of attributes provided in the context of online electronic commerce, Tan et al (2010) explicitly manipulate the number of alternatives provided, examining the effect of decision aids on the decision performance. Using resource-matching theory, the authors test impacts on decision performance of specific decision aids (low screening support, high screening support, and weight evaluation support) in low and high product attribute-load conditions. They show that "DSS can lead to greater information processing or effort minimization, depending on whether there is a match or mismatch in cognitive resources available to the consumers and those demanded in the task environment."

Karbing et al (2012) discuss the importance of conducting a retrospective evaluation of a DSS using archival data before conducting a prospective evaluation using live data. Quality of model fit can be evaluated with this retrospective evaluation in order to avoid in-use errors that might arise should the initial evaluation not be performed.

Phillips-Wren et al (2011) use a combined process-outcome DSS evaluation approach. They suggest that linking the DSS benefits to the DSS architecture provides a better measure of the underlying decision value to the individual user than evaluating either the decision-making process or the outcome of the process in isolation. The approach they consider is that suggested by Mora et al (2005), wherein a learning component is added to the intelligence, design, choice, and implementation decision phases first introduced by Simon (1960), and a four-level systems approach links the technical architecture of the DSS to the outcome and process elements at the top level. The authors use Analytic Hierarchy Process (AHP) to evaluate an intelligent DSS published by Lee (2004). They demonstrate how AHP allows for unique criteria weights assigned to the DSS components for each individual user, and that the user effectiveness is enhanced by the application of AHP to a combined process-outcome approach.

Using a simulation method, Ben-Zvi (2012) examines whether perceived DSS effectiveness is affected by six variables, including: familiarity with the system, perceived usefulness, use of system, perceived contribution to performance and success, user satisfaction, participation in defining the system, and system expectations being met. The results suggest that perceived DSS effectiveness is positively related to perceived usefulness, perceived contribution of the system, whether the system met users' expectations, and user satisfaction. However, no significant relationship is identified between system use, familiarity with the system, and participation in defining the system with perceived DSS effectiveness. The author concludes that researchers should be mindful of the importance of including certain subjective measures when examining the perceived effectiveness of a DSS. Additionally, the author indicates practitioners should be aware of the inverted U-shaped relationship between 
system complexity and performance identified by this study and others before it. At some point, a system can become too complex for users to be able to use them effectively.

In an experimental setting, Kayande et al (2009) examine whether model-driven DSS that facilitate the alignment of users' mental models with the system's recommendations are perceived as more valuable by the users. A dual-feedback DSS is designed to provide both feedback to help users correct their mental models and feedback about how much more could be gained by adjusting their mental model. The authors hypothesize that deep learning resulting in a relatively permanent change in someone's mental model would lead to a reduced cognitive dissonance and, therefore, improved DSS evaluations by users. Their findings suggest that, in order to encourage use, DSS should be designed to facilitate users' understanding of the basis for the DSS recommendations and why those recommendations will positively affect performance.

\section{BENEFITS}

Generally, usage of a DSS may result in a better decision process. That improved process may in turn create a better decision, but better decision quality may be difficult to document when working with semi-structured or unstructured problems. On the other hand, improvements to the decision process are often things more easily measured than decision quality. For example, we may be able to show that with a DSS, more alternatives are considered than without a DSS. Additionally, we may be able to show that post-DSS implementation, decisions are made in less time than prior to implementation. The DSS may serve to document a decision-making process and thereby raise confidence in a decision over and above what was possible without a DSS. It is possible that this higher level of confidence holds the benefit that it becomes easier to carry out the decision.

To provide specific illustration of DSS benefits, we will briefly discuss some decision support systems described in the literature. We will organize these examples according to the type of technology that drives the DSS. For each type, we will list at least one classic DSS well-known and long-known in the literature and generally several other systems that have been described in more recent years. The types of DSS we will include are communications-driven DSS, data-driven DSS, document-driven DSS, knowledge-driven DSS, model-driven DSS, and graphics-driven DSS. We have chosen to include these types of DDS because the examples illustrate a wide range of benefits arising from each driving technology. We are trying to show mostly recent efforts but also to illustrate the now-long history of the DSS field.

\section{Communications-driven DSS}

Communications-driven DSS are systems where the driving technology is a facilitation of interpersonal communications. Systems in this category are sometimes called Group Decision Support Systems (GDSS), Group Support Systems (GSS), or Groupware. These systems can benefit group decision-making by inhibiting negative group behaviors while promoting the positive aspects. Since negative aspects are more pronounced the larger the group, these systems provided the most benefit to large groups. Groupware (messaging, conferencing, group document handling, work flow, utilities/development tools, frameworks, services, and vertical market applications) facilitates movement of documents and messages to enhance the quality of communication among group members. Adding the DSS components (database management, model, and user interface) and, often a facilitator, the GDSS is able to provide process support in addition to decision-making support. Group memory aspects of the system allow retrospective analysis and interpretation of the process by group members. Other benefits afforded by GDSS include greater flexibility in the definition of meetings and anonymity of participation by group members as needed (Sauter, 2010).

A classic and widely-cited DSS in this category is described in Nunamaker et al (1989). In the context of a field study, the authors collect data about the process related to outcome effectiveness (quality of outcome and quality of session process), efficiency (costs and benefits relative to performing the same function manually), and user satisfaction (utilization rates and self-reported levels of satisfaction). With respect to effectiveness, they find that participation is more evenly distributed than would normally occur in a manual setting. Interviews with managers indicate they believe that the system provides outcome effectiveness as well. Regarding efficiency, man- 
hours are significantly reduced relative to manual settings. User satisfaction is strongly supported by utilization rates of the system, and this is confirmed by participants' self-reported levels of satisfaction.

More recently, Miller (2008) studies how the presence or absence of a facilitator affects the benefits of a GSS. Examining process facilitation from an Adaptive Structuration Theory perspective, the author compares highand low-levels of facilitator restrictiveness. The data indicate significantly different amounts and types of appropriation moves between the two levels of facilitator control. Additionally, the author examines the impact individual facilitators might have on the process. He finds substantial differences in both the quantity and the type of appropriation moves, and the proportion of moves involving the facilitator differ across facilitators as well.

Barkhi \& Kao (2010) use data envelopment analysis (DEA) to propose an economic efficiency measure for GDSS performance evaluation. The measure considers both decision inputs and outputs simultaneously, making it potentially more useful than traditional single-factor measures. Implementing a 2 by 2 by 2 factorial design through variation of three context variables (communication mode, group leadership mode, and incentive structure) in an experimental setting, the authors demonstrate that DEA can measure each GDSS user's performance and then determine how different contextual variables affect decision efficiency. Their results suggest that incentive structure and communication mode have a significant impact on DEA decision efficiency in the GDSS setting. They discuss possible extensions of their work including analyzing performance at the group level using redefined group inputs and outputs, and incorporating DEA methodology into development of next-generation GDSS.

Barkhi \& Kao (2011) extend their earlier work to examine the impact of psychological climate on GDSS users' decision-making performance. Their work specifically analyzes psychological safety, the freedom GDSS users believe they have to express themselves without negative impact, and psychological meaningfulness, which is the reciprocity felt by GDSS users in terms of returns from their input to the decision-making team. In lab settings where communication mode, incentive structure, and leadership mode were varied, they find that psychological safety does positively influence both GDSS users' task performance and work attitude. However, with respect to psychological meaningfulness, they find that it enhanced GDSS users' satisfaction with their participation but it is not significantly related to overall decision process efficiency.

Facilitating GDSS users in a distributed computing framework differs from the facilitation process in faceto-face settings. Computer-mediated communication must be used, which often results in a lower level of bonding among group participants. This lower bonding may subsequently cause participants to have lower interest and energy levels during meetings. Adla et al (2011) propose a set of tools that can be incorporated in the decisionmaking process to provide embedded facilitation to complement the facilitator's intervention effort. Dialogue Manager, Group Memory, Session Planning, and Group Toolkit modules comprise the distributed GDSS architecture. The Group Toolkit module consists of four categories: idea generation, idea organization, idea evaluation tools, and solution choice. The authors examine a three-phase group facilitation process (pre-meeting, during meeting, and post-meeting) in a case study at a plant that specializes in liquefying gas, providing a detailed discussion of the process activities as they can be enabled by the proposed GDSS toolkit.

\section{Data-driven DSS}

Data-driven DSS are those for which the underlying driving technology is a large stored databank. The earliest data-driven DSS of which we are aware is described by Donovan (1976). The system allows a decisionmaker to make ad hoc database inquiries to increase understanding in the area of energy policy. He provides a detailed example in order to show the importance of the interaction between an analytical system and a database system. Experience with the project demonstrates that an interactive system provides an advantage in terms of allowing quick changes to the model based on an unexpected data series being introduced. Elapsed time to see computational results is reduced, compared to earlier less-interactive systems. Overall, he demonstrates that time loss and transfer costs are lowered with an interactive system relative to database management systems in use at that time that were not integrated with the analytical system.

A more recent data-driven DSS was that of Jiang et al (1998) which combines a large amount of scanner data for a single consumer package goods category in supermarkets in a single metropolitan area with a time-series 
model for making both conditional and unconditional forecasts for a supermarket category. The benefit of the system is that it allows the decision-maker to model a forecast of a marketing policy under consideration including likely competitor reactions to a given competitive move. The system and its data and model increase brand managers' understanding of the consequences of an action under consideration.

Ferretti et al (2011) discuss ProgettoBosco, a data-driven DSS developed to optimize data collection related to Italian forests and forest management. Much of the knowledge resides in local forest managers, so one goal of the system is to integrate this knowledge base with the new technologies unfamiliar to many of them. Using a bottom-up approach of cooperation, successive approximations, and experimentation the authors develop a computer-based framework incorporating a DSS and a relational geodatabase. The DSS provides shared, standardized information at a national level, effective for all forest typologies in Italy. The user-friendly interface allows both forest managers and technical personnel to efficiently utilize the system.

\section{Document-driven DSS}

A document-driven DSS provides the decision-maker with relevant information coming from a store of documents. This category of DSS has arisen more recently than other categories such as data-driven or modeldriven DSS. Examples of document stores that could usefully drive decision-making processes would include news articles, user reviews or comments on websites, or emails. Also, many knowledge management systems such as Tiwana (2002) and Courtney (2001) are in this category. Such systems are often searchable collections of documents embodying "knowledge" submitted by members of a community of practice within an organization.

A recent document-driven DSS is described by Liu \& Lai (2011). The benefit of their system is to facilitate "knowledge reuse, cooperation and sharing among workers who perform similar ... tasks." Extending their previous work on mining an individual's knowledge flows (KFs), they develop an algorithm for mining and constructing a group KF. Using a dataset containing information about fourteen knowledge workers, the authors develop a prototype system to illustrate the algorithm proposed. The system performs worker cluster and group-based KF mining, omitting only the identification of knowledge-referencing paths phase of their proposed method. The benefits provided by group KF mining include facilitation of knowledge cooperation, sharing and reuse, as well as providing a useful reference for a novice when performing tasks.

\section{Knowledge-driven DSS}

Knowledge-driven DSS use a knowledge base extracted from the tacit knowledge of an expert. An early example of a knowledge-driven DSS is in Pan et al (1984). Such a system makes a knowledge base available for interaction with a decision-maker. It allows heuristics embedded in the knowledge base to be combined with algorithmic knowledge found in models and data found in a database to be combined with the decision-maker's insights. The difference between this type of system and a knowledge management system is that the knowledge in a knowledge-driven DSS is structured, often into a rule-base or some other heuristic mechanism.

Sklar et al (1990) describe a system that uses experts' knowledge coded in an expert system to build linear programming models. The resulting system is able to automate the model construction process, but it is up to the decision-maker to describe the problem to the system and then to execute the model and interpret the results. The benefit of the system is to incorporate experts' judgments into the process of constructing mathematical models and to speed up the model construction process.

Talluru \& Deshmukh (2003) use domain-specific knowledge to estimate costs for customized products. The benefit is in a more efficient bidding process. Using the system, engineers are able to estimate costs in 35 minutes for a bid that had required eight hours prior to implementation of the system. The system operates in a development environment, enabling engineers to manipulate requirements, and in a consulting environment, allowing sales personnel to prepare bids for jobs. The bidding process is carried out by the inference engine, consisting of a feature agent, steel cost agent, manufacturing cost agent and profit agent. Sales personnel work with the consulting environment to facilitate the bidding process. Feedback from both engineers and sales personnel following the initial testing phase is that is the system is useful, indicating it is both user-friendly and easy to update. 
Some problems are encountered thereafter, however, due to programmer turnover and lack of documentation. Additionally, the authors determine that explanation facilities would make the system more acceptable to users.

Waldburger (2011) discusses DeRISC (Dispute rEsolution Recommender for International Service Contracts), a knowledge-driven DSS, providing support for international service contracts between service providers and their customers. The system provides Private International Law (PIL)-compliant recommendations for contract parties concerning jurisdiction and applicable law. The purpose is to provide improved risk assessment for both contract parties throughout the service life cycle. The DSS implementation provides the first completely machineexecutable implementation of Brussels I regulation.

Wright et al (2009) discuss decision support capabilities included in commercially-available electronic medical record and computerized provider order entry systems. Generally, the DSS capability is a trigger that warns a provider when he/she is about to make a potentially dangerous order. These situations would include orders for drugs that a patient is allergic to or orders for drugs that have a harmful interaction with an already-prescribed drug. The decision support capability is advisory, and the provider has the ability to ignore the warning message. The realworld implications of these systems include reductions in the rate of adverse drug events.

\section{Model-driven DSS}

In a model-driven DSS, the driving technology is one or more mathematical model(s). Examples of types of models such a system might be built around include simulation models or the optimization models found in mathematical programming. The earliest model-driven DSS of which we are aware is described in Keen \& Morton (1978, pages 16-32). The system, which was initiated in 1966, supported production planning decisions for the laundry equipment division of a large American corporation. Before this system came into use, production planning took six days of effort spread over twenty elapsed days. Using the system, production planning took a half day spread over two elapsed days. It is clear the system allowed the planning process to take less effort and also to be more responsive. In addition, the system improved communications between marketing and production groups, allowed extended exploration of alternative production plans, and resulted in greater satisfaction with both the production planning process and with the resulting production plan.

Another classic example of a model-driven DSS is described in Seaberg \& Seaberg (1973). Their system consists of a family of models used for planning and control at Xerox of Canada. The models are executed in collaboration between computer-based models and functional managers. The models speed up planning and allow consideration of a larger number of cases than would be possible without the DSS. However, their models were not integrated, and output from one model would be manually input into the next.

Muhanna \& Pick (1994) discuss techniques to integrate free-standing models with each other and with databases. Their system is an approach to combine a series of simple models into a more-complex aggregate model. Their system allowed all data to be input only once and for output of a model to be subsequently used by another model. The benefit is in a reduction in both the labor and elapsed time required to build a model.

Chang (2002) outlines an example of a model-driven DSS. The primary benefit of her system is that it assists in model formulation of a staff scheduling problem. Models are built and exercised more quickly than would be possible without the DSS. Alternatively, the DSS causes better staff scheduling decisions than if the decisions would be made without the benefit of the optimization model. Audit schedulers must incorporate not only audit deadlines, but the sequence of sets of interrelated tasks. Additionally, efficiency variance based on different expertise levels of auditors must be considered. The proposed DSS explicitly considers each of those with the overall purpose of minimizing engagement completion time and, therefore, engagement cost. A pair-wise exchange model is used to improve the model's scheduling results. The author indicates the resulting method is especially suitable for large public accounting firms, and it is easy to use.

Xie (2010), based upon a European-Commission-funded project, discusses a model-driven DSS that integrates reliability analysis in the operation and service phases of a product's life cycle. The three-part conceptual model presented in the study uses Isograph, reliability engineering software, and ARENA, a system modeling 
package. Since ARENA is used primarily in supply chain management and manufacturing systems, incorporating its use in the DSS provides important linkage among the design, manufacturing, operation and service phases of the product life-cycle. Evaluating component and product reliability and maintenance through the life cycle, the model sees how new systems or features affect output.

\section{Graphics-driven DSS}

Graphics-driven DSS are primarily driven by their ability to display data meaningfully to decisionmaker(s). A very early graphics-driven DSS was the "Geodata Analysis and Display System (GADS)," Keen \& Morton (1978, pages 147-160), "GADS is an interactive graphics system that essentially draws maps." GADS has been used to plan for urban growth, design police beats, and modify school boundaries. Much of the benefit of the system seems to arise from the value of graphics in presenting data meaningfully and thereby assisting the decisionmaker in visual interpretation and focusing attention on the overall situation rather than isolated details.

Without actually describing an individual DSS, Desai (2005) outlines the use of visualization tools to enable geologists, geophysicists, and engineers in the oil industry to better understand their data and thereby come to better decisions about oil exploration and production. Integrating field operations data with data processing, analysis and interpretation has become much more efficient with the use of visualization centers, introduced in the late1990s. These centers also allow members of multidisciplinary teams to work collaboratively, viewing data simultaneously. The authors identify a large curved screen with multiple high resolution projectors, large flat screens, and caves ("small rooms where 3-D images are projected from outside") as the three primary 3-D visualization centers being used.

Tanerguclu et al (2012) discuss a graphics-based DSS that can be used in air defense to determine weapon and radar positions without having to travel to the locations being considered. The purpose of the system is to reduce the time required to select sites, using an optimization model incorporating needed theoretical assumptions and using reliable numerical data from a GIS. Additionally, the system is designed to be able to identify optimal deployment alternatives under changing conditions. The authors indicate that the spatial DSS can find an optimal solution to a more complicated problem in fewer than twenty minutes when visual analysis of a less complicated problem using a conventional paper map takes several hours on average. Moreover, without the use of a spatial DSS, it is often necessary to physically travel to the actual sites to check visibility of the point and feasibility of siting. Another benefit provided by the system is representation of results visually in addition to the solution reports.

\section{CONCLUSIONS}

The purposes of this paper were to introduce to the reader a sense of the power that DSS technologies bring to the decision-making process and to make them aware of the range of possible benefits from adopting DSS. This was accomplished by first outlining the evaluation of DSS for the purpose of assessing systems' benefits. After dividing the DSS literature into six parts according to the underlying driving technology, we then provided examples of DSSs from each area of the literature. In each area, we started with one of the very earliest systems of its type and then listed several more recent examples. The examples also come from a wide range of application domains. For each example, we discussed the benefits of the DSS.

\section{AUTHOR INFORMATION}

Roger Alan Pick is Professor of Management Information Systems at the Henry W. Bloch School of Management at the University of Missouri - Kansas City. He earned a Ph.D. from the Krannert Graduate School of Management at Purdue University in 1984. Pick's research interests are in decision support systems and management of information technology. His research on these topics has appeared in Communications of the ACM, Management Science, Journal of MIS, Decision Support Systems, IEEE Transactions, Information and Management, and numerous other outlets. These papers have been cited nearly 300 times. Contact: 816-235-2336, Henry W. Bloch School of Management, University of Missouri - Kansas City, 5110 Cherry Street, Kansas City, MO 64110-2499. E-mail: pickr@umkc.edu (Corresponding author) 
Nancy Weatherholt, C.P.A. is Associate Professor of Accounting at the Henry W. Bloch School of Management at the University of Missouri - Kansas City. She earned a Ph.D. from the University of Kansas in 1988. She has published articles in Behavioral Research in Accounting, Business Ethics Quarterly, The International Journal of Accounting, Asia-Pacific Journal of Accounting, Journal of Accountancy, Ohio CPA Journal, The Review of Accounting Information Systems, Internal Auditing, and Management Accounting. Contact: 816-235-2301, Henry W. Bloch School of Management, University of Missouri - Kansas City, 5110 Cherry Street, Kansas City, MO 64110-2499. E-mail: weatherholtn@umkc.edu

\section{REFERENCES}

1. $\quad$ Adelman, L. (1992). Evaluating Decision Support and Expert Systems. New York: John Wiley \& Sons.

2. $\quad$ Adla, A., Zarate, P., \& Soubie, J.-L. (2011). "A Proposal of Toolkit for GDSS Facilitators". Group Decision and Negotiation, 57-77.

3. Alic, I., Muntermann, J., \& Gregory, R. W. (2012). State of the Art of Financial Decision Support Systems based on Problem, Requirement, Component and Evaluation Categories. 25th Bled eConference eDependability: Reliable and Trustworthy eStructures, eProcesses, eOperations and eServices for the Future, (pp. 280-293).

4. Antunes, P., Herskovic, V., Ochoa, S. F., \& Pino, J. A. (2012). Structuring Dimensions for Collaborative Systems Evaluation. ACM Computing Surveys.

5. Barfod, M. B. (2012). Optimising Transport Decision-making using Customised Decision Models and Decision Conferences. Kongens Lyngby, Denmark: DTU Transport.

6. Barkhi, R., \& Kao, Y.-C. (2010). Evaluating Decision-making Performance in the GDSS Environment Using Data Envelopment Analysis. Decision Support Systems, 162-174.

7. Barkhi, R., \& Kao, Y.-C. (2011). Psychological Climate and Decision-Making Performance in a GDSS Context. Information \& Management, 125-134.

8. Ben-Zvi, T. (2012). Measuring the perceived effectiveness of decision support systems and their impact on performance. Decision Support Systems.

9. Chang, C. J. (2002). A Decision Support System for Audit Staff Scheduling of Multiple and Large-scaled Engagements. Review of Business Information Systems, 6(1), 27-40.

10. Courtney, J. F. (2001). Decision-making and Knowledge Management in Inquiring Organizations: A New Decision-making Paradigm for DSS. Decision Support Systems, 31(1), 17-38.

11. Defense, Department of. (2012, January 10). DAG Guidebook. Retrieved september 24, 2012, from at.dod.mil/docs/DefenseAcquisitionGuidebook.pdf

12. Desai, A. M. (2005). Group Collaboration and Group Decision-making Information Technologies in Petroleum Industry. Review of Business Information Systems, 9(3), 47-52.

13. Donovan, J. J. (1976). Database System Approach to Management Decision Support. ACM Transactions on Database Systems, 1(4), 344-369.

14. Ferretti, F., Dibari, C., DeMeo, I., Cantiani, P., \& Bianchi, M. (2011). A Data-Driven Decision Support System for Forest Planning. Mathematical and Computational Forestry \& Natural-Resource Sciences, 2735.

15. Finlay, P. N., \& Wilson, J. M. (1997). Validity of Decision Support Systems: Towards a Validation Methodology. Systems Research and Behavioral Science, 169-182.

16. Hallikainen, P., \& Chen, L. (2005). A Holistic Framework on Information Systems Evaluation with a Case Analysis. The Electronic Journal Information Systems Evaluation, 57-64.

17. Holsapple, C. W., \& Whinston, A. B. (1996). Decision Support Systems: A Knowledge-based Approach. Minneapolis/St. Paul: West.

18. Hosack, B., Hall, D., ,Paradice, D., \& Courtney, J. F. (2012, May). A Look Toward the Future: Decision Support Systems Research is Alive and Well. Journal of the Association for Information Systems, 13, 315340.

19. Jiang, J. J., Klein, G. J., \& Pick, R. A. (1998). A Marketing Category Management System: A Decision Support System Using Scanner Data. Decision Support Systems, 23(3), 259-271.

20. Jiggins, J. (1995). Development Impact Assessment: Impact Assessment of Aid Projects in Nonwestern Countries. Impact Assessment, 47-69. 
21. Karbing, D., Allerod, C., Thomsen, L., Espersen, K., Thorgaard, P., Andreassen, S., et al. (2012). Retrospective evaluation of a decision support system for controlled mechanical ventilation. Medical \& Biological Engineering \& Computing, 43-51.

22. Kayande, U., De Bruyn, A., Lilien, G. L., Rangaswamy, A., \& van Bruggen, G. H. (2009). How Incorporating Feedback Mechanisms in a DSS Affects DSS Evaluations. Information Systems Research, 527-546.

23. Kayande, U., De Bruyn, A., Lilien, G. L., Rangaswamy, A., \& van Bruggen, G. H. (2009). How Incorporating Feedback Mechanisms in a DSS Affects DSS Evaluations. Information Systems Research, 527-546.

24. Keen, P. G., \& Morton, M. S. (1978). Decision Support Systems: An Organizational Perspective. Reading, MA: Addison-Wesley.

25. Lee, W.-P. (2004). Applying Domain Knowledge and Social Information to Product Analysis and Recommendations: An Agent-Based Decision Support System. Expert Systems, 138-148.

26. Liu, D.-R., \& Lai, C.-H. (2011). Mining group-based knowledge flows for sharing task knowledge. Decision Support Systems, 50, 370-386.

27. Miah, S. J., Debuse, J., \& Kerr, D. (2012). A Development-Oriented DSS Evaluation Approach: A Case Demonstration for Conceptual Assessment". Australasian Journal of Information Systems, 43-55.

28. Miller, D. W. (2008). Facilitator Effects on the Process of GSS Appropriation: Opening the 'Black Box'. Review of Business Information Systems, 12(4), 57-75.

29. Mora, M., Forgionne, G., Garrido, F., Gupta, J., \& Gelman, O. (2005). Toward a Comprehensive Framework for the Design and Evaluation of Intelligent Decision-Making Support Systems (i-DMSS). Journal of Decision Systems, 321-344.

30. Muhanna, W. A., \& Pick, R. A. (1994). Meta-modeling Concepts and Tools for Model Management: A Systems Approach. Management Science, 40(9), 1093-1123.

31. Nunamaker, J., Vogel, D., Heminger, A., Martz, B., Growhowski, R., \& McGoff, C. (1989). Experience at IBM with group supportsystems: A field study. Decision Support Systems, 5(2), 183-196.

32. Pan, S., Pick, R. A., \& Whinston, A. B. (1984). A Formal Approach to Decision Support. In S. K. Chang, Management and Office Information Systems (pp. 91-118). New York: Plenum.

33. Patton, M. Q. (1982). Practical Evaluation. Newbury Park: Sage Publications, Inc.

34. Peffers, K., Tuunanen, T., Rothenberger, M., \& Chatterjeea, S. (2008). Design Science Research Methodology for IS Research. Journal of Management Information Systems, 45-77.

35. Petkova, O., \& Petkov, D. (2003). A Holistic Approach Towards the Validation and Legitimisation of Information Systems. Kybernetics, 703-714.

36. Petter, S., DeLone, W., \& McLean, E. R. (2012, May). The Past, Present, and Future of "IS Success". Journal of the Association for Information Systems, 13(5), 341-362.

37. Phillips-Wren, G., Mora, M., \& Forgionne, G. (2011). Assessing Today: Determining the Decision Value of Decision Support Systems. In S. (eds.), D. Paradice, F. Burstein, D. J. Power, \& R. Sharda, Decision Support, Annals of Information Systems 14 (pp. 203-220). Stillwater: Springer Science+Business Media, LLC.

38. Power, D. J. (2002). Decision Support Systems: Concepts and Resources for Managers. Westport, CT: Greenwood/Quorum Books.

39. Reason, J. (2008). The Human Contribution: Unsafe Acts, Accidents and Heroic Recoveries. Surrey: Ashgate.

40. Rennie, J. K., \& Singh, N. C. (1995, August 31). A guide for field projects on adaptive strategies. Retrieved october 2, 2012, from http://www.iisd.org/casl/caslguide/guidebook-home.htm

41. Rhee, C., \& Rao, H. R. (2008). Evaluation of Decision Support Systems. In F. Burstein, \& C. W. Holsapple, Handbook on Decision Support Systems, Volume 2 (pp. 313-327). Berlin: Springer.

42. Sauter, V. L. (2010). Decision Support Systems for Business Intelligence (2nd ed.). Hoboken, NJ: Wiley.

43. Scott, J. E. (1995). The Measurement of Information Systems Effectiveness: Evaluating a Measuring Instrument. Database: Advances in Information Systems, 43-59.

44. Seaberg, R. A., \& Seaberg, C. (1973). Computer Based Decision Systems in Xerox Corporate Planning. Management Science, 20(4), 575-584.

45. Simon, H. (1960). The New Science of Management Decision. New York: Harper and Row. 
46. Sklar, M., Pick, R. A., Vesprani, B., \& Evans, J. R. (1990). Eliciting Knowledge Representation Schema for Linear Programming. In D. E. Brown, \& C. C. White, Operations Research and Artificial Intelligence: The Integration of Problem Solving Strategies (pp. 279-316). Boston: Kluwer.

47. Talluru, L., \& Deshmukh, A. (2003). A Knowledge-based Decision Support System for Costing Job Orders. Review of Business Information Systems, 7(3), 1-12.

48. Tan, C.-H., Teo, H.-H., \& Benbasat, I. (2010). Assessing Screening and Evaluation Decision Support Systems: A Resource-Matching Approach. Information Systems Research, 305-326.

49. Tanerguclu, T., Maras, H., Gencer, C., \& Aygunes, H. (2012). A Decision Support System for Locating Weapon and Radar Positions in Stationary Point Air Defence. Information Systems Frontiers, 423-444.

50. Tiwana, A. (2002). The Knowledge Management Toolkit: Orchestrating IT, Strategy, and Knowledge Platforms (2nd Edition). Upper Saddle River, NJ: Prentice Hall.

51. Turban, E., Sharda, R., \& Delen, D. (2011). Decision Support Systems and Business Intelligence Systems (9th ed.). Upper Saddle River, New Jersey: Prentice Hall.

52. Waldburger, M. (2011). Decision Support in Contract Formation for Commercial Electronic Services with International Connection.

53. Wright, A., Sittig, D. F., Ash, J. S., Sharma, S., Pang, J. E., \& Middleton, B. (2009, September-October). Clinical Decision Support Capabilities of Commercially-available Clinical Information Systems. Journal of the American Medical Informatics Association, 16(5), 637-644.

54. Xie, C. (2010). A Model-Driven Decision Support System for Product Risk Analysis. Expert Systems, 388398.

NOTE: The first author of this paper published a paper titled "Benefits of Decision Support System" in the Handbook on Decision Support Systems (Burstein, Holsapple, eds.) published by Springer in 2008. Although covering a similar topic with a similar title, the present paper has little in common with the 2008 paper. The present paper uses a different organizing framework, and nearly all of the cited example systems are new. 
NOTES 